\title{
CHARACTERIZATIONS OF SPACES OF HOLOMORPHIC FUNCTIONS IN THE BALL
}

\author{
By Frank Beatrous, Jr. and Jacob Burbea
}

\begin{abstract}
Let $f$ be holomorphic in the unit ball of $\boldsymbol{C}^{n}$. Several equivalent criteria for $f$ to belong to the Hardy space $H^{p}$ as well as the weighted Bergman space $A_{q}^{p}, 0<p<\infty, q>0$, of the ball are established. In the one variable case, some of the above conditions reduce to those of Yamashita, characterizing Hardy spaces of the unit disk. In addition, various identities for the norm of $f$, in terms of a certain integrated counting function and certain Lusin characteristics, are obtained.
\end{abstract}

\section{$\S 1$. Introduction.}

The purpose of this paper is to give alternate characterizations of certain spaces of holomorphic functions in the unit ball $B$ of $C^{n}$. In particular, it will be shown that for $0<p<\infty$, a holomorphic function $f$ on $B$ is in the Hardy space $H^{p}(B)$ if and only if

$$
\int_{B} \partial_{\nu} \overline{\partial_{\nu}}|f|^{p}\left(1-\|z\|^{2}\right) d v(z)<\infty,
$$

where $\partial_{\nu}$ denotes radial derivative

$$
\partial_{\nu}=\sum_{j=1}^{n} z_{j} \partial_{j}
$$

In fact, this result will be obtained as a limiting case of a more general result on weighted Bergman spaces (Theorem 5.2). In the one variable case, condition (1.1) reduces to the condition of Yamashita [6] characterizing Hardy spaces of the unit disk.

In addition to the results alluded to above we also give characterizations of weighted Bergman and Hardy spaces in terms of an integrated counting function and in terms of certain Lusin conditions [2]. The present results extend previous results of Piranian and Rudin [3] and, more recently, of Yamashita [6] for the Hardy spaces of the unit disk. We also establish certain norm identities (Theorems 3.1 and 3.3) of the Hardy-Stein-Spencer type (see [1, p. 42] for the

Received February 2, 1984 
unit disk) in the unit ball $B$.

Section 2 contains the notation and the preliminaries of this paper. In section 3 we establish the above mentioned identities of the Hardy-Stein-Spencer type in $B$ (Theorems 3.1 and 3.3). Section 4 is devoted to a discussion on the Lusin property in the ball. An alternative expression for this property is described in Theorem 4.3. The main result of this paper is in section 5 (Theorem 5.2 and Corollary 5.3).

\section{§2. Preliminaries.}

Throughout this paper, $n$ will be a fixed integer, and $\boldsymbol{C}^{n}$ will be the vector space of ordered $n$-tuples $z=\left(z_{1}, \cdots, z_{n}\right)$ of complex numbers, with inner product and norm, given by

$$
\langle z, \zeta\rangle=\sum_{j=1}^{n} z_{j} \bar{\zeta}_{\jmath}, \quad\|z\|=\langle z, z\rangle^{1 / 2} .
$$

For $r>0, B(r) \equiv B_{n}(r)=\left\{z \in \boldsymbol{C}^{n}:\|z\|<r\right\}$ denotes the ball of radius $r$, centered at the origin, in $C^{n}$. The unit ball $B \equiv B_{n}$ in $C^{n}$ is then $B=B_{n}(1)$. The letter $v$ will stand for the Lebesgue measure of $C^{n}$ while $\sigma$ is the surface measure on the boundary $\partial B$ of $B$, normalized so that $\sigma(\partial B)=1$. The class of all holomorphic functions on $B$ will be denoted by $\mathcal{O}(B)$.

For $q>0$, we define

$$
d v_{q}(z) \equiv \frac{1}{\pi^{n}} \cdot \frac{\Gamma(n+q)}{\Gamma(q)}\left(1-\|z\|^{2}\right)^{q-1} d v(z) \quad(z \in B)
$$

which is a probability measure on $\bar{B}$. By an elementary calculation in polar coordinates one shows that the limit as $q \rightarrow 0^{+}$of $d v_{q}$ is

$$
d v_{0}(z)=d \sigma(z) \quad(z \in \partial B) .
$$

For $0<p<\infty$ and for $f \in \mathcal{O}(B)$, we define

$$
\begin{gathered}
M_{p}(r, f) \equiv\left\{\int_{\partial B}|f(r z)|^{p} d \sigma(z)\right\}^{1 / p} \quad(0 \leqq r<1), \\
\|f\|_{p} \equiv \sup \left\{M_{p}(r, f): 0 \leqq r<1\right\}
\end{gathered}
$$

and

$$
\|f\|_{p, q} \equiv\left\{\int_{B}|f(z)|^{p} d v_{q}(z)\right\}^{1 / p} \quad(q>0) .
$$

With these definitions we have

$$
\|f\|_{p, q}^{p}=\frac{2}{B(n, q)} \int_{0}^{1} r^{2 n-1}\left(1-r^{2}\right)^{q-1} M_{p}^{p}(r, f) d r,
$$

where $B(n, q)$ is the usual beta-function. Thus, by continuity, 


$$
\|f\|_{p}=\|f\|_{p, 0} \equiv \lim _{q \rightarrow 0^{+}}\|f\|_{p, q} .
$$

We may now define the weighted Bergman space

$$
A_{q}^{p}(B) \equiv\left\{f \in \mathcal{O}(B):\|f\|_{p, q}<\infty\right\} \quad(0<p<\infty, q \geqq 0) .
$$

Thus it follows that $A_{0}^{p}(B)$ is the ordinary $p$-Hardy space $H^{p}(B)$ and $A_{1}^{p}(B)$ is the ordinary p-Bergman space $A^{p}(B)$.

For $z=\left(z_{1}, \cdots, z_{n}\right) \in \boldsymbol{C}^{n}$, we write

$$
\partial_{\jmath} \equiv \frac{\partial}{\partial z_{\jmath}} \quad(1 \leqq \jmath \leqq n)
$$

for the partial complex derivatives, and

$$
\nabla \equiv\left(\partial_{1}, \cdots, \partial_{n}\right)
$$

for the complex-gradient. Moreover, the radial-derivative is defined as

$$
\partial_{\nu} \equiv\langle z, \bar{\nabla}\rangle=\sum_{j=1}^{n} z_{j} \partial_{\jmath} .
$$

It follows, in particular, that if $n=1$ and $f$ is holomorphic at $z \in C$, then $\partial_{\nu} f(z)$ $=z f^{\prime}(z)$.

Let $0<p<\infty$ and let $f \in \mathcal{O}(B)$. Then

$$
\partial_{\nu} \bar{\partial}_{\nu}|f|^{p}=\left(\frac{p}{2}\right)^{2}|f|^{p-2}\left|\partial_{\nu} f\right|^{2} .
$$

If, in addition, $f^{p / 2}$ is well-defined (i.e. either $p$ is an even integer or $f$ is free of zeros), then $\partial_{\nu} \bar{\partial}_{\nu}|f|^{p}=\left|\partial_{\nu} f^{p / 2}\right|^{2}$. On the other hand, the function

$$
f_{p}^{*}(z) \equiv \frac{p}{2}\|z\|^{-1}|f(z)|^{p / 2-1}\left|\partial_{\nu} f(z)\right|
$$

is well-defined (possibly infinite) and non-negative. This definition agrees with that of Yamashita [6] when $n=1$, and

$$
\left\{f_{p}^{*}(z)\right\}^{2}=\|z\|^{-2} \partial_{\nu} \bar{\partial}_{\nu}|f(z)|^{p} .
$$

For $0<p<\infty$, we define

$$
d \mu_{p}(\omega) \equiv \frac{1}{2 \pi}\left(\frac{p}{2}\right)^{2}|\omega|^{p-2} d m(\omega) \quad(\boldsymbol{\omega} \in \boldsymbol{C}),
$$

where $m$ is the Lebesgue area measure. Since $p>0, \mu_{p}$ is a rotationally invariant non-negative measure on $\boldsymbol{C}$ and belongs to $L_{\text {loc }}^{1}(\boldsymbol{C})$. Its Cauchy-transform is given by

$$
\hat{\mu}_{p}(\zeta) \equiv \lim _{\substack{\ell \rightarrow 0 \\ R \rightarrow \infty}} \int_{\varepsilon \leqq 1 \omega \mid \leqq R}(\zeta-\omega)^{-1} d \mu_{p}(\omega) \quad(\zeta \in C) .
$$

By expanding the integrand in geometric series in the disk $|\omega|<|\zeta|$ and in the 
annulus $|\zeta|<|\omega|<R$, and integrating term by term, we obtain

$$
\hat{\mu}_{p}(0)=0 ; \hat{\mu}_{p}(\zeta)=p|\zeta|^{p} / 4 \zeta \quad(\zeta \in C-\{0\}) .
$$

The unit disk $B_{1}$ in $C$ is denoted by $\Delta$ while $\Delta_{r}$ denotes the disk $B_{1}(r)$ of radius $r, r>0$. For $f \in \mathcal{O}(B)$ and $\zeta \in \partial B$, we define the slice function $f_{\zeta} \in \mathcal{O}(\Delta)$ by $f_{\zeta}(\lambda)=f(\lambda \zeta), \lambda \in \Delta$. Similarly, for $f \in \mathcal{O}(B)$ and $\lambda \in \Delta$, the dilation $f_{\lambda} \in \mathcal{O}(\bar{B})$ is defined by $f_{\lambda}(z)=f(\lambda z), z \in \bar{B}$. The apparent similar notation for these two different notions should cause no confusion.

Let $\boldsymbol{\omega} \in \boldsymbol{C}$ and $0 \leqq r \leqq 1$. For $f \in \mathcal{O}(\Delta)$, we let $n_{f}(r, \boldsymbol{\omega})$ be the number of zeros of the function $f(\cdot)-\omega$ in $\Delta_{r}$. If, on the other hand, $f \in \mathcal{O}(B), B=B_{n}$, we let

$$
n_{f}(r, \omega)=\int_{\partial B} n_{f \zeta}(r, \omega) d \sigma(\zeta),
$$

where $f_{\zeta}, \zeta \in \partial B$, is the slice function of $f$. We now use the integration by slices identity (see, for example, [4, p. 15]),

$$
\int_{\partial B} F(z) d \sigma(z)=\int_{\partial B} d \sigma(z) \cdot \frac{1}{2 \pi} \int_{0}^{2 \pi} F\left(e^{i \theta} z\right) d \theta,
$$

to prove :

Proposition 2.1. Let $\omega \in \boldsymbol{C}, 0 \leqq r<1$, and let $f \in \mathcal{O}(B)$. Then

$$
n_{f}(r, \omega)=\int_{\partial B} \frac{\partial_{\nu} f(r \zeta)}{f(r \zeta)-\omega} d \sigma(\zeta) .
$$

Proof. For $\zeta \in \partial B$, we consider the slice function $f_{\zeta}(\lambda)=f(\lambda \zeta), \lambda \in \Delta$. Thus $\lambda f_{\zeta}^{\prime}(\lambda)=\partial_{\nu} f_{\zeta}(\lambda)=\partial_{\nu} f(\lambda \zeta)$. Moreover, by definition

$$
\begin{aligned}
n_{f}(r, \omega) & =\int_{\partial B} d \sigma(\zeta) \cdot \frac{1}{2 \pi \imath} \int_{\mid \lambda !=r} \frac{f_{\zeta}^{\prime}(\lambda)}{f(\lambda \zeta)-\omega} d \lambda \\
& =\int_{\partial B} d \sigma(\zeta) \cdot \frac{1}{2 \pi} \int_{0}^{2 \pi} \frac{\partial_{\nu} f\left(r e^{i \theta} \zeta\right)}{f\left(r e^{i \theta} \zeta\right)-\omega} d \theta
\end{aligned}
$$

The result now follows from (2.12).

Let $0<p<\infty, 0 \leqq r<1$ and $f \in \mathcal{O}(B)$. We define

$$
N_{p}(r, f) \equiv \int_{C} n_{f}(r, \omega) d \mu_{p}(\omega) .
$$

It follows from (2.9)-(2.10) and Proposition 2.1 that

$$
N_{p}(r, f)=\int_{\partial B} \partial_{\nu} f(r \zeta) \hat{\mu}_{p}(f(r \zeta)) d \sigma(\zeta)=\frac{p}{4} \int_{\partial B} \frac{\partial_{\nu} f(r \zeta)}{f(r \zeta)}|f(r \zeta)|^{p} d \sigma(\zeta) .
$$

We denote by $\left\{e_{1}, \cdots, e_{n}\right\}$ and $\mathcal{U}=\mathcal{U}(n)$ the standard orthonormal basis and the group of all unitary transformations, respectively, of $\boldsymbol{C}^{n}$. Since $\sigma$ is U-invariant, we have (see $[4$, p. 15]) 


$$
\int_{\partial B} f(z) d \sigma(z)=\int_{U} f(U \zeta) d U
$$

where $\zeta$ is an arbitrary point of $\partial B$. In particular, $\zeta$ may be chosen to be $\zeta=e_{k}(k=1, \cdots, n)$. Another related identity is as follows : Let $f$ be a function of one complex variable. Then, an application of Fubini's theorem (see [4, p. 15]) shows that

$$
\int_{\partial B} f(\langle z, \zeta\rangle) d \sigma(z)=\frac{n-1}{\pi} \int_{\Delta}\left(1-|\omega|^{2}\right)^{n-2} f(\omega) d m(\omega)
$$

for any $\zeta \in \partial B \equiv \partial B_{n}$. This identity is also correct when $n=1$. The latter can be seen as a consequence of (2.1)-(2.2) or by letting $n \rightarrow 1$ and using l'Hôspital rule.

When $n=1$ we have the following triple identity due to Hardy, Stein and Spencer (see [1, p. 42]):

Proposition 2.2. Let $0<p<\infty, 0<r<1$ and $f \in \mathcal{O}(\Delta)$. Then

$$
\begin{aligned}
r \frac{d}{d r} M_{p}^{p}(r, f) & =\frac{p^{2}}{2 \pi} \int_{0}^{r} \rho d \rho \cdot \int_{0}^{2 \pi}\left|f\left(\rho e^{i \theta}\right)\right|^{p-2}\left|f^{\prime}\left(\rho e^{i \theta}\right)\right|^{2} d \theta \\
& =4 \int_{C} n_{f}(r, \omega) d \mu_{p}(\omega) .
\end{aligned}
$$

For $q \geqq 0$ and $0 \leqq t \leqq 1$, we introduce the non-negative functions of $t$ :

$$
I_{q}(t) \equiv I_{q}(t: n)=\frac{2}{B(n, q)} \int_{t}^{1} \rho^{2 n-1}\left(1-\rho^{2}\right)^{q-1} d \rho \quad(q>0)
$$

and

$$
J_{q}(t) \equiv J_{q}(t: n)=\frac{2}{B(n, q)} \int_{t}^{1} \rho^{2 n-1}\left(1-\rho^{2}\right)^{q-1} \log \frac{1}{\rho} d \rho \quad(q>0),
$$

and, by continuity

Thus

$$
I_{0}(t) \equiv 1, \quad J_{0}(t) \equiv 0
$$

and

$$
I_{q}(0)=1, \quad I_{q}(1)=J_{q}(1)=0 \quad(q>0)
$$

$$
J_{q}(0)=\frac{1}{2} \int_{0}^{1} x^{n-1} \frac{1-x^{q}}{1-x} d x .
$$

To prove the last relation, we write $\psi(s)$ for $\Gamma^{\prime}(s) / \Gamma(s), s>0$. Then, by $[5$, p. 262],

$$
\begin{aligned}
-2 B(n, q) J_{q}(0) & =\int_{0}^{1} \rho^{n-1}(1-\rho)^{q-1} \log \rho d \rho \\
& =\lim _{\varepsilon \rightarrow 0} \frac{1}{\varepsilon} \int_{0}^{1} \rho^{n-1}(1-\rho)^{q-1}\left(\rho^{\varepsilon}-1\right) d \rho
\end{aligned}
$$




$$
\begin{aligned}
& =\lim _{\varepsilon \rightarrow 0} \frac{1}{\varepsilon}\{B(n+\varepsilon, q)-B(n, q)\} \\
& =B(n, q)\{\psi(n)-\psi(n+q)\}=-B(n, q) \int_{0}^{1} x^{n-1} \frac{1-x^{q}}{1-x} d x,
\end{aligned}
$$

and (2.19) follows.

We now define

$$
K_{q}(t) \equiv K_{q}(t: n)=-\left\{(\log t) I_{q}(t)+J_{q}(t)\right\} \quad(q \geqq 0,0<t \leqq 1) .
$$

Thus, by (2.17)-(2.18),

$$
K_{q}(t)=\frac{2}{B(n, q)} \int_{0}^{1} \rho^{2 n-1}\left(1-\rho^{2}\right)^{q-1} \log \frac{\rho}{t} d \rho .
$$

The following proposition is obtained by applying l'Hôspital's rule to the above definitions.

Proposition 2.3. Let $q \geqq 0$. Then

$$
\begin{aligned}
& \lim _{t \rightarrow 1}(1-t)^{-q} I_{q}(t)=2^{q} \frac{\Gamma(n+q)}{\Gamma(n) \Gamma(q+1)}, \\
& \lim _{t \rightarrow 1}(1-t)^{-(q+1)} J_{q}(t)=2^{q} \frac{q \Gamma(n+q)}{\Gamma(n) \Gamma(q+2)}
\end{aligned}
$$

and

$$
\lim _{t \rightarrow 1}(1-t)^{-(q+1)} K_{q}(t)=2^{q} \frac{\Gamma(n+q)}{\Gamma(n) \Gamma(q+2)} .
$$

\section{$\S 3$. Hardy-Stein-Spencer Type Inequalities on the Ball.}

The following theorem constitutes an extension of the identities of Hardy, Stein and Spencer in Proposition 2.2 to the ball $B=B_{n}$.

THEOREM 3.1. Let $f \in \mathcal{O}(B), 0<p<\infty$ and $0<r<1$. Then

$$
\begin{aligned}
r \frac{d}{d r} M_{p}^{p}(r, f) & =\frac{2 \Gamma(n)}{\pi^{n}} \int_{B(r)}\left\{f_{p}^{*}(z)\right\}^{2}\|z\|^{-2(n-1)} d v(z) \\
& =4 N_{p}(r, f) \\
& =p \int_{\partial B} \frac{\partial_{\nu} f(r \zeta)}{f(r \zeta)}|f(r \zeta)|^{p} d \sigma(\zeta) .
\end{aligned}
$$

Proof. For a fixed $\zeta \in \partial B$ we consider the slice function $f_{\zeta}(\lambda)=f(\lambda \zeta), \quad \lambda \in \Delta$. Thus $f_{\zeta} \in \mathcal{O}(\Delta)$ and

$$
\lambda f_{\zeta}^{\prime}(\lambda)=\partial_{\nu} f_{\zeta}(\lambda)=\partial_{\nu} f(\lambda \zeta) \quad(\lambda \in \Delta) .
$$

We now apply Proposition 2.2 to the function $f_{\zeta}$ and express the result in terms of $f$. This gives 


$$
\begin{aligned}
r \frac{d}{d r} & \cdot \frac{1}{2 \pi} \int_{0}^{2 \pi}\left|f\left(r e^{i \theta} \zeta\right)\right|^{p} d \theta \\
& =p^{2} \int_{0}^{r} \rho^{-1} d \rho \cdot \frac{1}{2 \pi} \int_{0}^{2 \pi}\left|f\left(\rho e^{i \theta} \zeta\right)\right|^{p-2}\left|\partial_{\nu} f\left(\rho e^{i \theta} \zeta\right)\right|^{2} d \theta \\
& =4 \int_{c} n_{f \zeta}(r, \omega) d \mu_{p}(\omega) .
\end{aligned}
$$

Letting $\zeta$ now vary, we integrate the last identities with respect to $\sigma$, and use the integration by slices identity (2.12) and Fubini's theorem. The theorem follows now at once by using (2.3), (2.8)-(2.11) and (2.13)-(2.14).

As a consequence of this theorem we obtain the following result. For $a>0$, we use the standard notation of $\log ^{+} a$ to stand for $\max (\log a, 0)$ and $\chi_{A}$ for the characteristic function of a set $A$.

Corollary 3.2. Let $f \in \mathcal{O}(B), 0<p<\infty$ and $0<r<1$. Then

$$
\begin{aligned}
M_{p}^{p}(r, f)-|f(0)|^{p} & =\frac{2 \Gamma(n)}{\pi^{n}} \int_{B}\left\{f_{p}^{*}(z)\right\}^{2}\|z\|^{-2(n-1)} \log ^{+} \frac{r}{\|z\|} d v(z) \\
& =4 \int_{0}^{r} \rho^{-1} N_{p}(\rho, f) d \rho \\
& =\frac{\Gamma(n)}{2 \pi^{n}} p \int_{B(z)} \frac{\partial_{\nu} f(z)}{f(z)}|f(z)|^{p}\|z\|^{-2 n} d v(z) .
\end{aligned}
$$

Proof. Integrating the first identity of Theorem 3.1 and using Fubini's theorem, we obtain

$$
M_{p}^{p}(r, f)-|f(0)|^{p}=\frac{2 \Gamma(n)}{\pi^{n}} \int_{B}\left[\int_{0}^{r} t^{-1} \chi_{B(t)}(z) d t\right]\left\{f_{p}^{*}(z)\right\}^{2}\|z\|^{-2(n-1)} d v(z) .
$$

Now,

and thus

$$
\chi_{B(t)}(z) \equiv \chi_{(\|z\|, 1)}(t)
$$

$$
\begin{aligned}
\int_{0}^{r} t^{-1} \chi_{B(t)}(z) d t & =\int_{0}^{r} t^{-1} \chi_{(\|z\|, 1)}(t) d t \\
& =\int_{0}^{1} t^{-1} \chi_{(\|z\|, 1) \cap(0, r)}(t) d t \\
& =\log ^{+} \frac{r}{\|z\|} .
\end{aligned}
$$

This gives the first identity of the corollary. The second identity is an immediate consequence of the second identity of Theorem 3.1. As for the third identity, we integrate the third identity of Theorem 3.1. This gives

$$
M_{p}^{p}(r, f)-|f(0)|^{p}=p \int_{0}^{r} \rho^{2 n-1} \rho^{-2 n} d \rho \cdot \int_{\partial B} \frac{\partial_{\nu} f(\rho \zeta)}{f(\rho \zeta)}|f(\rho \zeta)|^{p} d \sigma(\zeta)
$$




$$
=\frac{\Gamma(n)}{2 \pi^{n}} p \int_{B(r)} \frac{\partial_{\nu} f(z)}{f(z)}|f(z)|^{p}\|z\|^{-2 n} d v(z)
$$

and the third identity of the corollary follows. This concludes the proof.

The last corollary, coupled with the notation of (2.1)-(2.7) and (2.17)-(2.21) yields the following result which is of interest on its own right.

THEOREM 3.3. Let $f \in A_{q}^{p}(B)$ with $0<p<\infty$ and $q \geqq 0$. Then

$$
\begin{aligned}
\|f\|_{p, q}^{p}-|f(0)|^{p} & =\frac{2 \Gamma(n)}{\pi^{n}} \int_{B}\left\{f_{p}^{*}(z)\right\}^{2}\|z\|^{-2(n-1)} K_{q}(\|z\|) d v(z) \\
& =4 \int_{0}^{1} \rho^{-1} N_{p}(\rho, f) I_{q}(\rho) d \rho \\
& =\frac{\Gamma(n)}{2 \pi^{n}} p \int_{B} \frac{\partial_{\nu} f(z)}{f(z)}|f(z)|^{p}\|z\|^{-2 n} I_{q}(\|z\|) d v(z) .
\end{aligned}
$$

In particular, when $q=0$, i.e. when $f \in H^{p}(B) \equiv A_{0}^{p}(B)$, then

$$
\begin{aligned}
\|f\|_{p}^{p}-|f(0)|^{p} & =\frac{2 \Gamma(n)}{\pi^{n}} \int_{B}\left\{f_{p}^{*}(z)\right\}^{2}\|z\|^{-2(n-1)} \log \frac{1}{\|z\|} d v(z) \\
& =4 \int_{0}^{1} \rho^{-1} N_{p}(\rho, f) d \rho \\
& =\frac{\Gamma(n)}{2 \pi^{n}} p \int_{B} \frac{\partial_{\nu} f(z)}{f(z)}|f(z)|^{p}\|z\|^{-2 n} d v(z) .
\end{aligned}
$$

Proof. The particular case of $q=0$ is an immediate consequence of Corollary 3.2 and (2.3)-(2.4). The more general case of $q \geqq 0$ is obtained from Corollary 3.2, (2.6), (2.17)-(2.21) and Fubini's theorem, and by noting that

$$
2 \int_{0}^{1} r^{2 n-1}\left(1-r^{2}\right)^{q-1} \log ^{+} \frac{r}{\|z\|} d r=B(n, q) K_{q}(\|z\|) \quad(z \in B-\{0\})
$$

and that, for $\rho=\|z\|, z \in B$,

$$
2 \int_{0}^{1} \chi_{(0, r)}(\rho) r^{2 n-1}\left(1-r^{2}\right)^{q-1} d r=2 \int_{0}^{1} \chi_{B(r)}(z) r^{2 n-1}\left(1-r^{2}\right)^{q-1} d r=B(n, q) I_{q}(\rho) .
$$

The proof is now complete.

\section{$\S 4$. The Lusin Property in the Ball.}

For $\zeta \in \partial B$ we denote by $D_{\alpha}(\zeta), \alpha>0$, the region

$$
D_{\alpha}(\zeta)=\left\{z \in C^{n}:|1-\langle z, \zeta\rangle|<\frac{\alpha}{2}\left(1-\|z\|^{2}\right)\right\} .
$$

When $\alpha \leqq 1, D_{\alpha}(\zeta)$ is the empty set while for $\alpha>1, D_{\alpha}(\zeta)$ is the customary 
Korány approach region (see $[4$, p. 72$]$ ) and is a subdomain of $B$. Moreover, $D_{\alpha}(\zeta)$ is monotonically increasing to $B$ as $\alpha \rightarrow \infty$. Evidently,

$$
U\left(D_{\alpha}(\zeta)\right)=D_{\alpha}(U \zeta) \quad(U \in \mathcal{U}) .
$$

Let $\alpha>1,0<p<\infty$ and $q \geqq 0$. For $f \in \mathcal{O}(B)$ and $\zeta \in \partial B$, we define

$$
L_{p}(\zeta: q ; f ; \alpha) \equiv \int_{D_{\alpha}(\zeta)}\left\{f_{p}^{*}(z)\right\}^{2}\left(1-\|z\|^{2}\right)^{q+1-n} d v(z)
$$

and

$$
\mathcal{L}_{p}(f: q, \alpha) \equiv \int_{\partial B} L_{p}(\zeta: q ; f ; \alpha) d \sigma(\zeta) .
$$

We say that $f$ has the $(p, q)$-Lusin property with respect to $\alpha$ if $\mathcal{L}_{p}(f: q, \alpha)<\infty$. The $U$-invariance of this property can be read off from the following proposition:

Proposition 4.1. Let $\alpha>1,0<p<\infty$ and $q \geqq 0$. Then for $f \in \mathcal{O}(B)$ we have:

$$
\mathcal{L}_{p}(f: q, \alpha)=\int_{U} L_{p}\left(U \zeta_{0}: q ; f ; \alpha\right) d U
$$

where $\zeta_{0}$ is an arbutrary point of $\partial B$. Moreover, for any $\zeta \in \partial B$ and any $U \in \mathcal{U}$, we have

$$
L_{p}(U \zeta: q ; f ; \alpha)=L_{p}(\zeta: q ; f \circ U ; \alpha) .
$$

Proof. The first identity is an immediate consequence of the definition (4.3) and the identity (2.15). As for the second identity, we first prove that

$$
\partial_{\nu} f(U \omega)=\partial_{\nu}(f \circ U)(\omega)
$$

for any $\omega \in B$ and any $U \in \mathcal{U}$. Indeed, for $1 \leqq \jmath \leqq n$ and $z=U \omega \in B$ the chain-rule gives

and thus

$$
\begin{aligned}
\partial_{\jmath} f(U \omega) & =\left\langle\nabla(f \circ U)(\omega), \overline{\partial_{j} U^{*} z}\right\rangle=\left\langle\nabla(f \circ U)(\omega), \bar{U}^{*} e_{\jmath}\right\rangle \\
& =\left\langle\bar{U} \nabla(f \circ U)(\omega), e_{\jmath}\right\rangle=[\bar{U} \nabla(f \circ U)(\omega)]_{\jmath},
\end{aligned}
$$

It follows that

$$
\nabla f(U \omega)=\bar{U} \nabla(f \circ U)(\omega) .
$$

$$
\begin{aligned}
\partial_{\nu} f(U \omega) & =\langle\nabla f(U \omega), \overline{U \omega}\rangle=\langle\bar{U} \nabla(f \circ U)(\omega), \bar{U} \bar{\omega}\rangle \\
& =\langle\nabla(f \circ U)(\omega), \bar{\omega}\rangle=\partial_{\nu}(f \circ U)(\omega)
\end{aligned}
$$

and (4.4) is proved. Now, from (4.4) and (2.8) we deduce that

$$
f_{p}^{*}(U \omega)=(f \circ U)_{p}^{*}(\omega) \quad(\omega \in B, U \in \mathcal{U}) .
$$

It follows from (4.1)-(4.2) that for any $\zeta \in \partial B$ and any $U \in \mathcal{U}$, 
This concludes the proof.

$$
\begin{aligned}
L_{p}(U \zeta: q ; f ; \alpha) & =\int_{U\left(D_{\alpha}(\zeta)\right)}\left\{f_{p}^{*}(z)\right\}^{2}\left(1-\|z\|^{2}\right)^{q+1-n} d v(z) \\
& =\int_{D_{\alpha}(\zeta)}\left\{f_{p}^{*}(U \omega)\right\}^{2}\left(1-\|U \omega\|^{2}\right)^{q+1-n} d v(U \omega) \\
& =\int_{D_{\alpha}(\zeta)}\left\{(f \circ U)_{p}^{*}(\omega)\right\}^{2}\left(1-\|\omega\|^{2}\right)^{q+1-n} d v(\omega) \\
& =L_{p}(\zeta: q ; f \circ U ; \alpha) .
\end{aligned}
$$

For any $\alpha>1$ and $z \in B$, we define

$$
Q_{\alpha}(z) \equiv\left\{\zeta \in \partial B:|1-\langle z, \zeta\rangle|<\frac{\alpha}{2}\left(1-\|z\|^{2}\right)\right\}
$$

and

$$
\omega_{\alpha}(z) \equiv \sigma\left(Q_{\alpha}(z)\right)
$$

We note that

$$
\chi_{D_{\alpha}(\zeta)}(z)=\chi_{Q_{\alpha}(z)}(\zeta) \quad(z \in B, \zeta \in \partial B)
$$

and, of course,

In particular,

$$
U\left(Q_{\alpha}(z)\right)=Q_{\alpha}(U z) \quad(U \in U) .
$$

$$
\omega_{\alpha}(U z)=\omega_{\alpha}(z) \quad(z \in B, U \in \mathcal{U}) .
$$

For $z \in B$, we choose a $U_{z} \in \mathcal{U}$ with $U_{z} z=\|z\| e_{1}$, and thus $\omega_{\alpha}(z)=\omega_{\alpha}\left(\|z\| e_{1}\right)$. It follows that $\omega_{\alpha}$ is a non-negative radially-symmetric function on $B$. Accordingly, we have

$$
F_{\alpha}(r) \equiv \omega_{\alpha}(z)=\omega_{\alpha}\left(r e_{1}\right)=\sigma\left(Q_{\alpha}\left(r e_{1}\right)\right) \quad(r=\|z\|, z \in B) .
$$

This quantity is essentially proportional to $\left(1-r^{2}\right)^{n}$. In fact, we shall prove:

PROPOSITION 4.2. For $\alpha>1$, the function $G_{\alpha}(r: n) \equiv F_{\alpha}(r) r^{2(n-1)}\left(1-r^{2}\right)^{-n}$ increases from 0 to a finite positive limat $G_{\alpha}(1: n)$ as $r$ increases from $|\alpha-2| / \alpha$ to 1. Moreover.

and

$$
G_{\alpha}(1: 1)=\left(\alpha^{2}-1\right)^{1 / 2} / \alpha \pi
$$

$$
G_{\alpha}(1: n)=\frac{n-1}{2 \pi}\left(\frac{\alpha}{2}\right)^{n-2} \int_{1}^{\alpha}(t-1)^{n-1}\left(\alpha^{2}-t^{2}\right)^{1 / 2} d t \quad(n \geqq 2) .
$$

In particular, $G_{\alpha}(1: n) \leqq \pi^{-1}[\alpha(\alpha-1) / 2]^{n-1}$ for every $n \geqq 1$.

Proof. By (4.8),

$$
F_{\alpha}(r)=\int_{\partial B} \chi_{Q_{\alpha}\left(r e_{1}\right)}(\zeta) d \sigma(\zeta)
$$


Here $\chi_{Q_{\alpha}\left(r e_{1}\right)}(\zeta) \equiv g\left(\left\langle e_{1}, \zeta\right\rangle\right)$ where $g$ is a function of one complex variable $\lambda$ satisfying $g(\lambda)=1$ if $|1-r \lambda|<\alpha\left(1-r^{2}\right) / 2$ and $g(\lambda)=0$ otherwise. It follows that $F_{\alpha}(r)>0$ for $|\alpha-2| / \alpha<r<1$ and $F_{\alpha}(|\alpha-2| / \alpha)=0$. Moreover, an application of the identity (2.16) to the present function $g$ gives

$$
F_{\alpha}(r)=\frac{n-1}{\pi} \int_{E_{\alpha}(r)}\left(1-|\lambda|^{2}\right)^{n-2} d m(\lambda)
$$

where

$$
E_{\alpha}(r)=\left\{\lambda \in \Delta:|1-r \lambda|<\frac{\alpha}{2}\left(1-r^{2}\right)\right\} \quad(|\alpha-2| / \alpha<r<1) .
$$

We note that the set $E_{\alpha}(r)$ is empty for $r \leqq|\alpha-2| / \alpha$. Also, the case of $n=1$ is simpler and may also be obtained by letting $n \rightarrow 1$ in the expression for $F_{\alpha}(r)$. The change of variable

$$
\frac{1}{r}-\lambda=\frac{1}{\omega} \cdot \frac{\alpha}{2} \cdot \frac{1-r^{2}}{r}
$$

constitutes a conformal mapping of $E_{\alpha}(r)$ onto the region $E_{\alpha}^{*}(r)$ given by

$$
E_{\alpha}^{*}(r)=\left\{\omega \in C:|\omega|>1,\left|\omega-\frac{\alpha}{2}\right|<r \frac{\alpha}{2}\right\} \quad(|\alpha-2| / \alpha<r<1) .
$$

It follows that

$$
F_{\alpha}(r)=\frac{n-1}{\pi}\left(\frac{\alpha}{2}\right)^{2} \frac{\left(1-r^{2}\right)^{n}}{r^{2(n-1)}} \int_{E_{\alpha}^{*}(r)}\left\{r^{2}\left(\frac{\alpha}{2}\right)^{2}-\left|\omega-\frac{\alpha}{2}\right|^{2}\right\}^{n-2}|\omega|^{-2 n} d m(\omega)
$$

and hence

$$
G_{\alpha}(r: n)=\frac{n-1}{\pi}\left(\frac{\alpha}{2}\right)^{2} \int_{E_{\alpha}^{*}(r)}\left\{r^{2}\left(\frac{\alpha}{2}\right)^{2}-\left|\omega-\frac{\alpha}{2}\right|^{2}\right\}^{n-2}|\omega|^{-2 n} d m(\omega) .
$$

As $r$ increases, both the integrand and the domain of integration $E_{\alpha}^{*}(r)$ of the last integral increase. It follows from the monotone convergence theorem that the limit of $G_{\alpha}(r: n)$, as $r \rightarrow 1$, exists and equals

$$
G_{\alpha}(1: n)=\frac{n-1}{\pi}\left(\frac{\alpha}{2}\right)^{2} \int_{E_{\alpha}^{*}(1)}\left\{\left(\frac{\alpha}{2}\right)^{2}-\left|\omega-\frac{\alpha}{2}\right|^{2}\right\}^{n-2}|\omega|^{-2 n} d m(\omega) .
$$

The proposition now follows by a routine calculation of the last integral.

The following theorem gives another characterization of the Lusin property.

THEOREM 4.3. Let $\alpha>1,0<p<\infty, q \geqq 0$ and $|\alpha-2| / \alpha<r_{0}<1$. Then for any $f \in \mathcal{O}(B)$ we have

$$
\begin{array}{r}
G_{\alpha}\left(r_{0}\right) \int_{A\left(r_{0}\right)}\left\{f_{p}^{*}(z)\right\}^{2}\left(1-\|z\|^{2}\right)^{q+1}\|z\|^{-2(n-1)} d v(z) \leqq \mathcal{L}_{p}(f: q, \alpha) \\
\leqq G_{\alpha}(1) \int_{A(|\alpha-2| / \alpha)}\left\{f_{p}^{*}(z)\right\}^{2}\left(1-\|z\|^{2}\right)^{q+1}\|z\|^{-2(n-1)} d v(z),
\end{array}
$$


where $G_{\alpha}(r) \equiv G_{\alpha}(r: n)$ and $A(r) \equiv\{z \in B:\|z\|>r\},|\alpha-2| / \alpha \leqq r \leqq 1$.

Proof. By (4.2)-(4.3), Fubini's theorem and (4.5)-(4.7)

$$
\begin{aligned}
\mathcal{L}_{p}(f: q, \alpha) & =\int_{B}\left[\int_{\partial B} \chi_{D_{\alpha}(\zeta)}(z) d \sigma(\zeta)\right]\left\{f_{p}^{*}(z)\right\}^{2}\left(1-\|z\|^{2}\right)^{q+1-n} d v(z) \\
& =\int_{B} \omega_{\alpha}(z)\left\{f_{p}^{*}(z)\right\}^{2}\left(1-\|z\|^{2}\right)^{q+1-n} d v(z),
\end{aligned}
$$

and thus, by (4.8),

$$
\mathcal{L}_{p}(f: q, \alpha)=\int_{B}\left\{f_{p}^{*}(z)\right\}^{2}\left(1-\|z\|^{2}\right)^{q+1-n} F_{\alpha}(\|z\|) d v(z) .
$$

The theorem now follows from Proposition 4.2 and by observing that $F_{\alpha}(\|z\|)=0$ for $\|z\| \leqq|\alpha-2| / \alpha$.

Let $\alpha>1,0<p<\infty$ and let $f \in \mathcal{O}(B)$. We say that $f$ has the $p$-Lusin property with respect to $\alpha$ if $f$ has the $(p, 0)$-Lusin property with respect to $\alpha$. When $n=1$, this definition reduces to that of Piranian and Rudin [3] for $p=2$, and to that of Yamashita [6] for any $0<p<\infty$.

\section{$\S 5$. Criteria for the Space $A_{q}^{p}(B)$.}

We shall now prove the main results mentioned in the introduction of this paper. Before proceeding, however, we shall prepare the following lemma:

LEMMA 5.1. Let $0<p<\infty, q \geqq 0$ and let $f \in \mathcal{O}(B)$. Then

$$
\int_{B(r)}\left\{f_{p}^{*}(z)\right\}^{2}\|z\|^{-2(n-1)} K_{q}(\|z\|) d v(z)<\infty
$$

and

$$
\int_{0}^{r} \rho^{-1} N_{p}(\rho, f) I_{q}(\rho) d \rho<\infty
$$

for every $0 \leqq r<1$.

Proof. For any $0 \leqq r<1$, the dilation $f_{r}$ is in $A_{q}^{p}(B)$. It follows from Theorem 3.3 that

$$
\begin{aligned}
\infty & >\pi^{n}\left(\left\|f_{r}\right\|_{p, q}^{p}-\left.f(0)\right|^{p}\right) / 2 \Gamma(n) \\
& =\int_{B}\left\{\left(f_{r}\right)_{p}^{*}(z)\right\}^{2}\|z\|^{-2(n-1)} K_{q}(\|z\|) d v(z) \\
& =\frac{2 \pi^{n}}{\Gamma(n)} \int_{0}^{1} \rho^{-1} N_{p}\left(\rho, f_{r}\right) I_{q}(\rho) d \rho .
\end{aligned}
$$

However, by (2.8),

$$
\left(f_{r}\right)_{p}^{*}(z)=r f_{p}^{*}(r z) .
$$

Similarly, by Proposition 2.1 and (2.13) 


$$
N_{p}\left(\rho, f_{r}\right)=N_{p}(r \rho, f) \text {. }
$$

Thus, by the change of variables $\omega=r z$ and $t=r \rho$,

$$
\begin{aligned}
\infty & >\pi^{n}\left(\left\|f_{r}\right\|_{p, q}^{p}-|f(0)|^{p}\right) / 2 \Gamma(n) \\
& =\int_{B(r)}\left\{f_{p}^{*}(\omega)\right\}^{2}\|\omega\|^{-2(n-1)} K_{q}\left(\frac{\|\omega\|}{r}\right) d v(\omega) \\
& =\frac{2 \pi^{n}}{\Gamma(n)} \int_{0}^{r} t^{-1} N_{p}(t, f) I_{q}\left(\frac{t}{r}\right) d t .
\end{aligned}
$$

It follows that

$$
\int_{B(r)}\left\{f_{p}^{*}(z)\right\}^{2}\|z\|^{-2(n-1)} K_{q}\left(\frac{\|z\|}{r}\right) d v(z)<\infty
$$

and

$$
\int_{0}^{r} \rho^{-1} N_{p}(\rho, f) I_{q}\left(\frac{\rho}{r}\right) d \rho<\infty
$$

for every $0<r<1$. We now show that

$$
\int_{B(s)}\left\{f_{p}^{*}(z)\right\}^{2}\|z\|^{-2(n-1)} d v(z)<\infty
$$

for every $0<s<1$. It is sufficient to show this for $1 / 2 \leqq s<1$. In this case, we let $r=(1+s) / 2$ and thus $1 / 2 \leqq s<r$. Then, since by $(2.21) K_{q}$ is a positive and decreasing function on $(0,1)$, we obtain from (5.1)

$$
\begin{aligned}
& \infty>\int_{B(r)}\left\{f_{p}^{*}(z)\right\}^{2}\|z\|^{-2(n-1)} K_{q}\left(\frac{\|z\|}{r}\right) d v(z) \\
& \geqq\left.\int_{B(s)}\left\{f_{p}^{*}(z)\right\}^{2}\|z\|^{-2(n-1)}\right) K_{q}\left(\frac{\|z\|}{r}\right) d v(z) \\
& \geqq K_{q}\left(\frac{2 s}{1+s}\right) \int_{B(s)}\left\{f_{p}^{*}(z)\right\}^{2}\|z\|^{-2(n-1)} d v(z) .
\end{aligned}
$$

This proves (5.3). In a similar manner, since by (2.17) $I_{q}$ is a positive and decreasing function on $(0,1)$, we obtain from (5.2) that

$$
\int_{0}^{s} \rho^{-1} N_{p}(\rho, f) d \rho<\infty
$$

for every $0<s<1$. Let now $z \in B(r), 0<r<1$. Then, by $(2.21)$.

$$
\begin{aligned}
K_{q}(\|z\|)-K_{q}\left(\frac{\|z\|}{r}\right)=\frac{2}{B(n, q)}\left\{\int_{\|z\|}^{\|z\| / r} \rho^{2 n-1}\left(1-\rho^{2}\right)^{q-1} \log \frac{\rho}{\|z\|} d \rho\right. \\
\left.\quad+\log \frac{1}{r} \int_{\|z\| / r}^{1} \rho^{2 n-1}\left(1-\rho^{2}\right)^{q-1} d \rho\right\}
\end{aligned}
$$




$$
\leqq\left(\log \frac{1}{r}\right) \frac{2}{B(n, q)} \int_{\|z\|}^{1} \rho^{2 n-1}\left(1-\rho^{2}\right)^{q-1} d \rho \leqq \log \frac{1}{r}
$$

or

$$
K_{q}(\|z\|) \leqq K_{q}\left(\frac{\|z\|}{r}\right)+\log \frac{1}{r} \quad(z \in B(r), 0<r<1) .
$$

This, together with (5.1) and (5.3) gives the first assertion of the lemma. The second assertion follows in a similar fashion. Indeed, by (2.17),

and thus

$$
\begin{aligned}
I_{q}(\rho)-I_{q}\left(\frac{\rho}{r}\right) & =\frac{2}{B(n, q)} \int_{\rho}^{\rho / r} t^{2 n-1}\left(1-t^{2}\right)^{q-1} d t \\
& \leqq \frac{2}{B(n, q)} \int_{t}^{1} t^{2 n-1}\left(1-t^{2}\right)^{q-1} d t=1,
\end{aligned}
$$

$$
I_{q}(\rho) \leqq I_{q}\left(\frac{\rho}{r}\right)+1 \quad(0 \leqq \rho<r<1) .
$$

Again, this with (5.2) and (5.4) establishes the second assertion of the lemma, and the proof is complete.

THEOREM 5.2. Let $0<p<\infty, q \geqq 0$ and $f \in \mathcal{O}(B)$. Then the following statements are equivalent:

(i) $\|f\|_{p, q}<\infty$, i.e. $f$ is in the class $A_{q}^{p}(B)$;

(ii) $\int_{0}^{1} N_{p}(\rho, f)\left(1-\rho^{2}\right)^{q} d \rho<\infty$;

(iii) $\int_{B}\left\{f_{p}^{*}(z)\right\}^{2}\left(1-\|z\|^{2}\right)^{q+1} d v(z)<\infty$;

(iv) $\mathcal{L}_{p}(f ; q, \alpha)<\infty$ for every $\alpha>1$, i.e. $f$ has the $(p, q)$-Lusin property with respect to every $\alpha>1$;

(v) $\mathcal{L}_{p}(f ; q, \alpha)<\infty$ for some $\alpha>1$, i.e. $f$ has the $(p, q)$-Lusin property with respect to some $\alpha>1$.

Proof. We shall show that (i) $\Leftrightarrow($ ii), (i) $\Leftrightarrow($ iii $) \Rightarrow($ iv $) \Rightarrow(v) \Rightarrow($ iii $)$.

(i) $\Leftrightarrow($ ii) : By Theorem 3.3, (i) is equivalent to

$$
\int_{0}^{1} \rho^{-1} N_{p}(\rho, f) I_{q}(\rho) d \rho<\infty .
$$

On the other hand, by Proposition 2.3 there exist an $r_{0} \in(0,1)$ and constants $c_{j}=c_{j}\left(r_{0}\right)>0(j=1,2)$ such that

$$
c_{1}\left(1-\rho^{2}\right)^{q} \leqq I_{q}(\rho) \leqq c_{2}\left(1-\rho^{2}\right)^{q} \quad\left(r_{0}<\rho<1\right) .
$$

Moreover, by Lemma 5.1,

$$
\int_{0}^{r_{0}} \rho^{-1} N_{p}(\rho, f) I_{q}(\rho) d \rho<\infty
$$


and thus

In particular,

$$
\int_{0}^{r_{0}} N_{p}(\rho, f) d \rho<\infty
$$

$$
\int_{0}^{r_{0}} N_{p}(\rho, f)\left(1-\rho^{2}\right)^{q} d \rho \leqq \int_{0}^{r_{0}} N_{p}(\rho, f) d \rho<\infty .
$$

Using (5.6)-(5.8) we find that (5.5) is equivalent to (ii), and the assertion follows. (i) $\Leftrightarrow$ (iii): As before, by Theorem 3.3, (i) is equivalent to

$$
\int_{B}\left\{f_{p}^{*}(z)\right\}^{2}\|z\|^{-2(n-1)} K_{q}(\|z\|) d v(z)<\infty,
$$

and by Proposition 2.3 there exist $r_{0} \in(0,1)$ and $c_{j}=c_{j}\left(r_{0}\right)>0(j=1,2)$ such that

$$
c_{1}\left(1-\|z\|^{2}\right)^{q+1} \leqq K_{q}(\|z\|) \leqq c_{2}\left(1-\|z\|^{2}\right)^{q+1} \quad\left(r_{0}<\|z\|<1\right) .
$$

Again, by Lemma 5.1,

$$
\int_{B\left(r_{0}\right)}\left\{f_{p}^{*}(z)\right\}^{2}\|z\|^{-2(n-1)} K_{q}(\|z\|) d v(z)<\infty
$$

and thus

$$
\int_{B\left(r_{0}\right)}\left\{f_{p}^{*}(z)\right\}^{2} d v(z)<\infty
$$

In particular,

$$
\int_{B\left(r_{0}\right)}\left\{f_{p}^{*}(z)\right\}^{2}\left(1-\|z\|^{2}\right)^{q+1} d v(z)<\infty .
$$

Therefore, by (5.10)-(5.12), (5.9) is equivalent to (iii), and the assertion follows. (iii) $\Rightarrow$ (iv): This follows from Theorem 4.3, (2.21) and Lemma 5.1. (iv) $\Rightarrow(\mathrm{v})$ : This is a triviality.

$(\mathrm{v}) \Rightarrow$ (iii): This follows from Theorem 4.3, (2.21) and Lemma 5.1.

Letting $q=0$ in this theorem gives the following result which was alluded to in the introduction.

Corollary 5.3. Let $0<p<\infty$ and $f \in \mathcal{O}(B)$. Then the following statements are equivalent:

(i) $\|f\|_{p}<\infty$, i.e. $f$ is in the class $H^{p}(B)$;

(ii) $\int_{0}^{1} N_{p}(\rho, f) d \rho<\infty$;

(iii) $\int_{B}\left\{f_{p}^{*}(z)\right\}^{2}\left(1-\|z\|^{2}\right) d v(z)<\infty$;

(iv) $\mathcal{L}_{p}(f: 0, \alpha)<\infty$ for every $\alpha>1$, i.e. $f$ has the $p$-Lusin property with respect to every $\alpha>1$; 
(v) $\mathcal{L}_{p}(f: 0, \alpha)<\infty$ for some $\alpha>1$, i.e. $f$ has the $p$-Lusin property with respect to some $\alpha>1$.

When $n=1$, the equivalence of statements (i), (iii), (iv) and ( $\mathrm{v}$ ) of this corollary was proved, by using different methods, in Piranian and Rudin [3] for the case of $p=2$ and in Yamashita [6] for the more general case of $0<p<\infty$.

It should also be noted that statement (iii) of Theorem 5.2 may be replaced by $\int_{B}\left\{f_{p}^{*}(z)\right\}^{2}\|z\|^{2 k}\left(1-\|z\|^{2}\right)^{q+1} d v(z)<\infty$ for any $k \geqq-(n-1)$. Indeed, for any such $k$ the integrand is locally integrable on $B$ by virtue of (5.3). Thus the convergence of divergence of this integral is determined by the behaviour of the integrand near $\partial B$. In the particular, $q=0$ and $k=2$, gives, by using (2.8), condition (1.1) of the introduction, which is equivalent to statement (iii) of Corollary 5.3.

\section{REFERENCES}

[1] Hayman, W. K., Multivalent Functions, Cambridge Unıv. Press, London, 1958.

[2] Lusin, N., Sur une properiété des functions a carré sommable, Bull. Calcutta Math. Soc., 20 (1930), 139-154.

[3] Piranian, G. AND W. Rudin, Lusin's theorem on areas of conformal maps, Michigan Math. J., 3 (1955-1956), 191-199.

[4] Rudin, W., Function Theory in the Unit Ball of $\boldsymbol{C}^{n}$, Springer-Verlag, New York, 1980.

[5] Whittaker, E. T. and G. N. Watson, A course of modern analysis, Cambridge Univ. Press, London, 1973.

[6] Yamashita, S., Criteria for functions to be of Hardy class $H^{p}$, Proc. Amer. Math. Soc., 75 (1979), 69-72.

Department of Mathematics

University of PitTsburgh

Pittsburgh, Pensylvania 15260

U.S.A. 